\title{
Vibrational Collapse of Hexapod Packings
}

\author{
Yuchen Zhao ${ }^{1, \star}$, Jingqiu Ding ${ }^{2}$, Jonathan Barés ${ }^{3}, H u$ Zheng ${ }^{1}$, Karola Dierichs ${ }^{4}$, Achim Menges ${ }^{4}$, and Robert \\ Behringer ${ }^{1}$ \\ ${ }^{1}$ Department of Physics and Center for Nonlinear and Complex Systems, Duke University, Durham, NC, USA. \\ ${ }^{2}$ Department of Physics, Nanjing University, Nanjing, China. \\ ${ }^{3}$ Université de Montpellier, Montpellier, France. \\ ${ }^{4}$ Institute for Computational Design, University of Stuttgart, Stuttgart, Germany
}

\begin{abstract}
Columns made of convex noncohesive grains like sand collapse after being released from a confining container. However, structures built from non-convex grains can be stable without external support. In the current experiments, we investigate the effect of vibration on destroying such columns. The change of column height during vertical vibration, can be well characterized by stretched exponential relaxation when the column is short, which is in agreement with previous work, while a faster collapse happens when the column is tall. We investigate the collapse after the fast process including its dependence on column geometry, and on interparticle and basal friction.
\end{abstract}

\section{Introduction}

Jamming of granular matter is a wide-spread phenomenon $[1,2]$. One can see piles of sand and rocks, packing of apples and oranges in grocery stores. The jamming of soft, frictionless spheres has been studied in a detail [2], and yet new ways of jamming emerge constantly [3, 4]. One of the recent explorations is the jamming of non-convex particles [5-11]. In a typical experiment, if one prepares a packing of the particles in a vertical tube and then removes the tube confinement, the cylindrical packing can retain its shape without confinement, and possibly sustain compression and shear [10]. This new phenomenon is not only interesting from the packing and jamming theory point of view [12-15], but also has significant implications, such as material and architectural design [16-18]. Open questions include: what are the physics that causes this jamming and how can one quantitatively describe the effect of particle shapes on the ability to jam and design new materials for applications based on particle level properties [19]. In particular, the work by Gravish et. al. [8] on staple packings is relevant here. Their experiment involves collapse of a column under external vibration. During collapse, the centroid height of the column follows a stretched exponential relation: $h(t)=h(0) \exp \left[-(t / \tau)^{\beta}\right]$ [20]. Further, the entanglement between particles was quantified. It is interesting to ask whether the packing of other extreme shaped particles has a similar response to vibration.

Here we studied the collapse of columns made with hexapod (star-shaped) particles under vibration of the base supporting the column (Fig.1). In the first part of the paper, we present our experimental setup, preparation and data

\footnotetext{
^e-mail: yz172@phy.duke.edu
}

characterizing the collapse of hexapod columns. In the second part, we confirm the applicability of a stretched exponential relation for collapse of hexapod columns under vibration. Although a stretched exponential describes the collapse dynamics where the column height is low enough, we observe deviation from this relation when the initial centroid height of the column is large enough. We also studied the effect of interparticle and basal friction on the stretched exponential relation. The final section provides a discussion and conclusions.
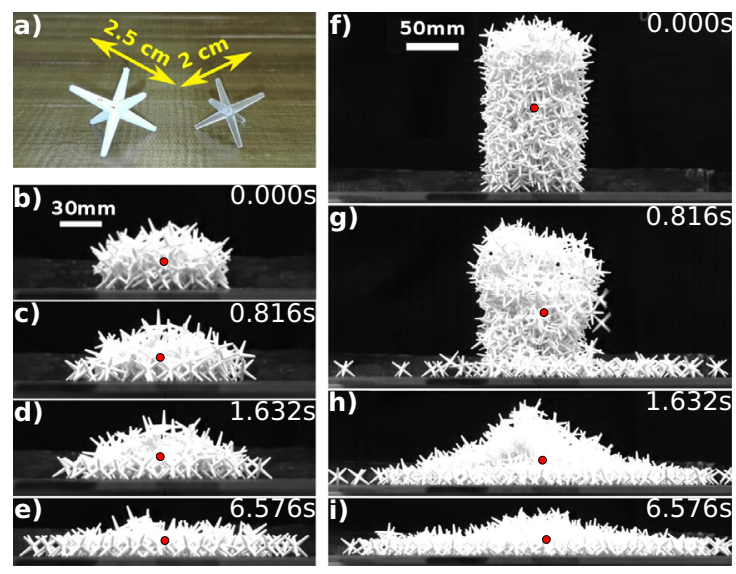

Figure 1. (a) Nylon (left) and acrylic (right) particles and snapshots from two trials ((b)-(e) and (f)-(i)) in N1. The centroid height of the packing $h(t)$ are measured and marked as a red dot in each image. (b)-(e): collapse of a column with $h(0)=11.6 \mathrm{~mm}$. (f)-(i): collapse of a column with $h(0)=71.4 \mathrm{~mm}$. Both columns were prepared with $D=102 \mathrm{~mm}$ and sat on a Teflon sheet base with low friction coefficient. A sinusoidal oscillation with frequency $f=58.6 \pm 0.3 \mathrm{~Hz}$ and $\Gamma=2.0 \pm 0.2$ was applied. 


\section{Experimental Set-up}

We used two kinds of hexapods (Fig.1 (a)), which are the same as those used in [10]. Each has the same hexapod shape: six equal-length arms with square cross-section lying symmetrically in the xyz direction. One kind is made of cast acrylic, which has a low friction coefficient, $0.4 \pm 0.1$, between any pair of particle arms. The other is made of laser-sintered white nylon PA2200, and has friction coefficient $1.0 \pm 0.3$. The arm tip-to-tip length is $s=20 \mathrm{~mm}$ and $s=25 \mathrm{~mm}$ for the acrylic and nylon hexapods, respectively. In the following we refer to the acrylic particles as low friction (LF) particles, and the nylon high friction (HF). Columns were prepared by first randomly pouring particles into a straight PVC tube (inner diameter $D$ ) from $40 \mathrm{~cm}$ above the base. The process was the same as [10]. Each tube was $30 \mathrm{~cm}$ tall. Then the tube was lifted vertically without disturbing the packing. The tube rested on either a Teflon surface, which had a friction coefficient $0.3 \pm 0.1$ between particles and the surface, or a sand paper surface, which had a higher friction coefficient such that particles only rolled when we tilted the surface. We refer to the Teflon and sand paper as low friction (LF) and high friction (HF) bases.

We prepared columns with different diameters $D$ and initial heights, which were chosen to be around the critical height $h_{c}$ in our previous results [10]. Columns were placed on a vibration plate and we applied sinusoidal vibrations to induce collapse (See Fig.2). The sinusoidal vibration had frequency $f=58.6 \pm 0.3 \mathrm{~Hz}$ and dimensionless vibration strength $\Gamma=A \omega^{2} / g=2.0 \pm 0.2$, which were kept unchanged in all runs. On top of the plate, we glued either a Teflon sheet or sand paper as described earlier.

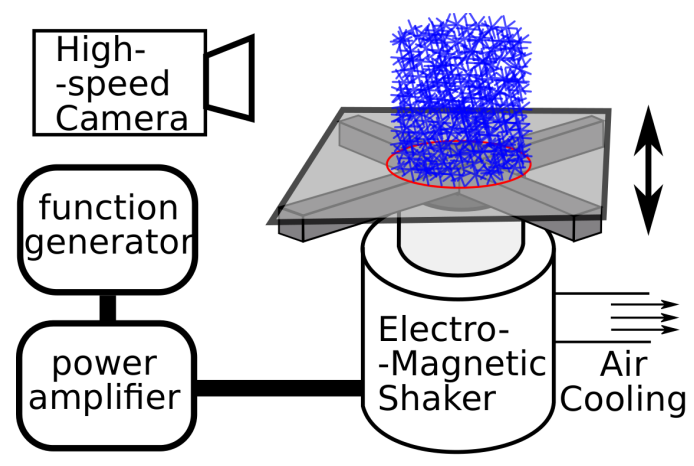

Figure 2. Schematic of the setup: The vibration generator consisted of an electromagnetic shaker that drove a $45 \mathrm{~cm}$-by- 45 $\mathrm{cm}$ square plate, that was screwed to a pair of square, aluminum tubes that strengthen the base. The driving sinusoidal signal was generated by a function generator, which was then amplified by a power amplifier. The shaker was cooled by air. We measured the vibration amplitude by imaging the vibrating plate via a highspeed camera (Photron FASTCAM SA5). The first peak of the vibration was large relative to later vibration, but contributed insignificantly to the packing compared to the total amount of energy from the vibration.

All the collapse dynamics were captured by high speed video imaging at $250 \mathrm{frame} / \mathrm{s}$ on the LF base and 60 frame/s on the HF base. Homemade Python codes were used to post-process the images and the centroid height $h(t)$ of the packing in the images was found (Fig.1). Because the packings were almost axially symmetric, the centroid height was close to the center of mass of the packing. We summarized the experiments in Table.1.

\section{Results}

\subsection{Relaxation dynamics}

In Fig.1, we present several representative snapshots during column collapses on the LF base. Fig.1 (b) to (e) describes the collapse process of an initially short column relative to its width. Particles on the edge of the column fell off within the first second, and formed a cone shape (Fig.1(c)). Then, the cone shortened and widened slowly until there was almost a single layer of particles on the vibrating plate. Fig.1(f)-(i) shows that a column with large $h(0)$ collapsed differently. It took about 1.6 second for the column to gradually shorten and widen as in Fig.1(f) and (g). After a cone was formed, the packing collapsed in a similar way as the initially short column. We also observed this difference with LF hexapod, although the first of the two-step process happened faster than HF, tall columns (data not shown).

In Fig.3, we present the collapse dynamics of short columns and test the assumption of a stretched exponential relation for both HF and LF hexapods, and two different column diameters. By plotting the dimensionless centroid height $h(t) / h(0)$ as $-\ln [h(t) / h(0)]$ vs. $t$. on a log$\log$ scale, the assumption $h(t)=h(0) \exp \left[-(t / \tau)^{\beta}\right]$ would be shown as a straight line. We found these collapses are well approximated by stretched exponentials. The exponent $\beta$ is between 0.5 and 1 , which is similar to previous work by Gravish et. al. [8]. However, the dependence of $\beta$ on initial diameter or particle friction properties are not clear here. Pictorially, these collapse processes resemble Fig.1(b)-(e). For HF hexapods, the collapse dynamics from two different diameters overlap within errorbars. The situation is not clear for LF hexapods. The characteristic relaxation times are $\tau=3.0 \mathrm{~s}$ and $\tau=1.3 \mathrm{~s}$ for $\mathrm{HF}$ and LF hexapods, respectively. These results agree with our previous study [10] showing that columns made of HF hexapod are more stable than LF ones.

\subsection{Deviation from relaxation dynamics}

As we increased $h(0)$, however, we observed that the dynamics of $h(t) / h(0)$ significantly deviated from a stretched exponential relation. As in Fig.4(a), the data are well fitted to a straight line for low $h(0)$, but gradually bend near time $T_{b}$ as the initial centroid height increases, and the collapse dynamics depends on $h(0)$. Before $T_{b}$, collapse is relatively fast, corresponding to a massive falling of those particles which constitute the top or middle of the column, while the bottom of the column retains its original shape (Fig.1(g)). After $T_{b}$, particles formed a cone-shaped pile and then slowly relaxed under vibration (Fig.1(h,i)) as for 
Table 1. Parameters used for vibration-collapse experiments: particle and base friction properties, column geometry.

\begin{tabular}{lllllll}
\hline $\mathcal{N}$ & Hexapod & Bases & $D(\mathrm{~mm})$ & $h(t=0)(\mathrm{mm})$ & $D / s$ & $h(t=0) / s$ \\
\hline 1 & HF & LF & 102,130 & $12 \rightarrow 72$ & $4.1,5.2$ & $0.48 \rightarrow 2.9$ \\
2 & LF & LF & 102,130 & $7 \rightarrow 62$ & $5.1,6.5$ & $0.35 \rightarrow 3.1$ \\
3 & HF & HF & 102 & $31 \rightarrow 78$ & 4.1 & $1.2 \rightarrow 2.9$ \\
4 & LF & HF & 102 & $14 \rightarrow 66$ & 5.1 & $0.7 \rightarrow 3.3$ \\
\hline
\end{tabular}

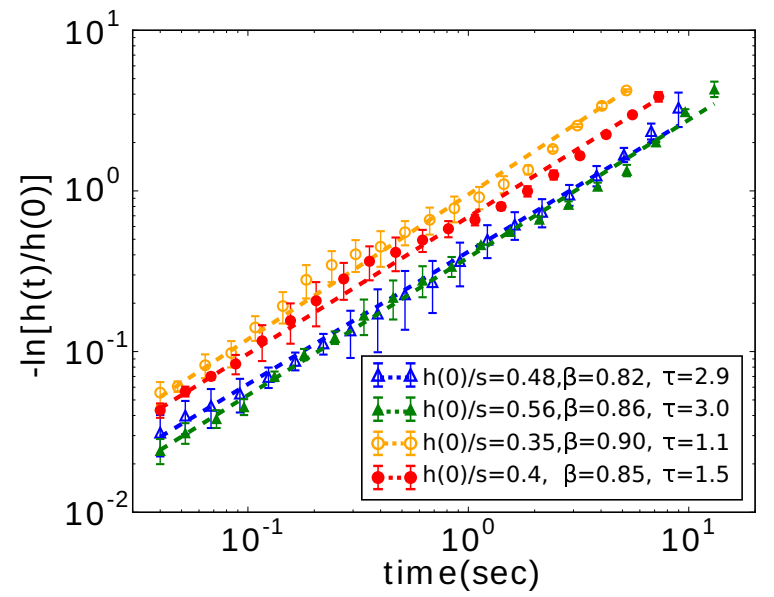

Figure 3. The negative $\ln []$ of dimensionless centroid height $h(t) / h(0)$ vs. time $t$ on a $\log -\log$ scale. Data from $\mathcal{N} 1$ and $\mathcal{N} 2$. Markers: triangle for frictional particles and circle for frictionless particles; filled symbols for $D=130 \mathrm{~mm}$ and empty symbols for $D=102 \mathrm{~mm}$. Each data curve is an average of 2 or 3 repeated independent trials, with the errorbars indicate variation between trials. For fitting, we take 20 data points evenly spaced on the logarithmic time scale, and fit them into a straight line with the form: $y(x)=\beta x-\beta \ln (\tau)$ which minimizes the least square error. The fitting parameters are in the legend of the plot, and the unit of $\tau$ is second. The change of number of points used in the fitting does not change only slightly change the fitting parameters but not our conclusions.

short columns. We identify these bending points $T_{b}$ to be roughly the point where the cone-shape pile was formed and particles stopped rolling down from the top. One such snapshot at $T_{b}$ is Fig.1(h). Such a two-step process happens for LF hexapods, as well as both hexapods on the HF base (not shown).

We do not have a specific form for the collapse before reaching $T_{b}$. For data after the bending point, we took $T_{b}$ and $h\left(T_{b}\right)$ at the bending point to be the new initial condition for the collapse, and fitted to a stretched exponential relation. The assumption behind shifting the starting point of the relaxation is that, there are two distinct collapse processes before and after $T_{b}$. We observed experimentally that the collapse of a cone-shape pile (Fig.1 (b-e,h and i)) could be stopped immediately after the vibration was turned off, while the collapse of taller columns could not (Fig.1f and g, and it is also true for LF hexapod experiments). After $T_{b}$, the pile configurations were more stable than the column. Our assumption is supported by the data shown in Fig.4(b) for experiments N1. In Fig.4(b) we fit the dynamics after $T_{b}$ for curve $h(0) / s=2.9$. The choice
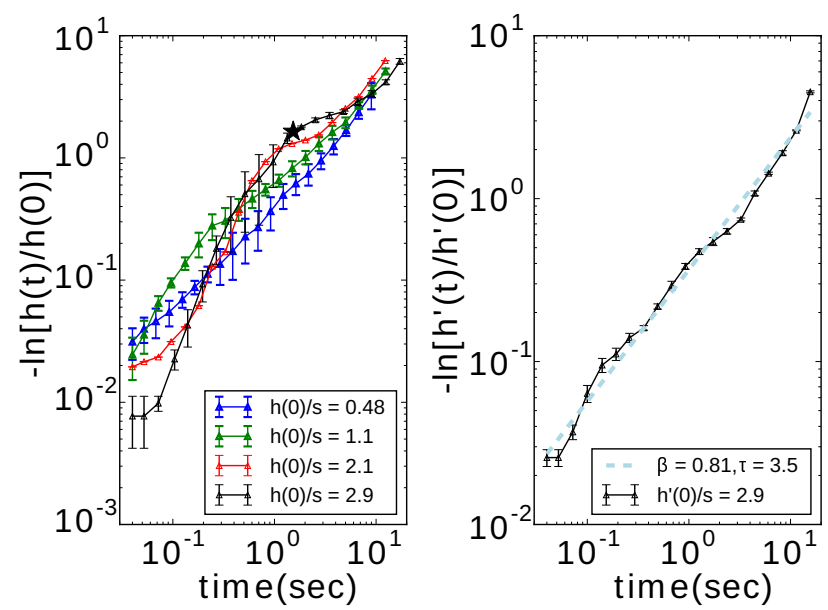

Figure 4. (a): Relaxation of $h(t)$ for columns with various $h(0)$ and diameter $D=102 \mathrm{~mm}$. Each data is an average of 2-3 independent, repeated trial. The data for $h(0) / s=0.48$ is the same one used in Fig.3. The black star on the data $h(0) / s=2.9$ indicates the $T_{b}$, whose snapshot is shown in Fig.1(g). (b): Dynamics after the $T_{b}$ for the $h(0)=$ data. Formally, We define $h^{\prime}\left(t^{\prime}\right)=h\left(t^{\prime}+T_{b}\right),\left(t^{\prime}>=0\right)$ and fit a straight line into $-\ln \left[h^{\prime}\left(t^{\prime}\right) / h^{\prime}(0)\right]$ vs $t^{\prime}$ on a log-log scale, as in Fig.3.

of $T_{b}$ here corresponds to the moment when the cone was formed. We obtain similar fitting parameters as for the short columns ( $\beta=0.8$ and $\tau=3.0 \mathrm{~s}$ ) (Fig.3), which indicates the collapse of the column after $T_{b}$ likely shares similar mechanisms as the collapse of short columns.

\subsection{Effect of friction}

Our assumption is further supported by experiments $\mathcal{N} 3$, e.g. HF particles on HF base, shown in Fig.5 and insert. In the insert, the whole collapse dynamics are presented. The separation between fast collapse and slow relaxation is clear, and more apparent with increasing $h(0)$, which agrees with the experiments on LF base. We define new time zeros at $T_{b}$ and fit the data to stretched exponentials, which are shown in the main figure. Overall, the good fits suggest that the collapse processes for cone-shape piles can again be approximated by stretched exponentials on the HF base. One difference is, that the characteristic relaxation times $\tau$ are 10 times larger than the ones for LF base, and the exponent $\beta$ is systematically smaller, around 0.5 to 0.6 . We attribute the increase of relaxation time to drag with the frictional base. A packing relaxes by expanding in the horizontal directions. On the frictional base, the horizontal expansion is retarded. Thus, under the same external vibration, the relaxation is slower. 


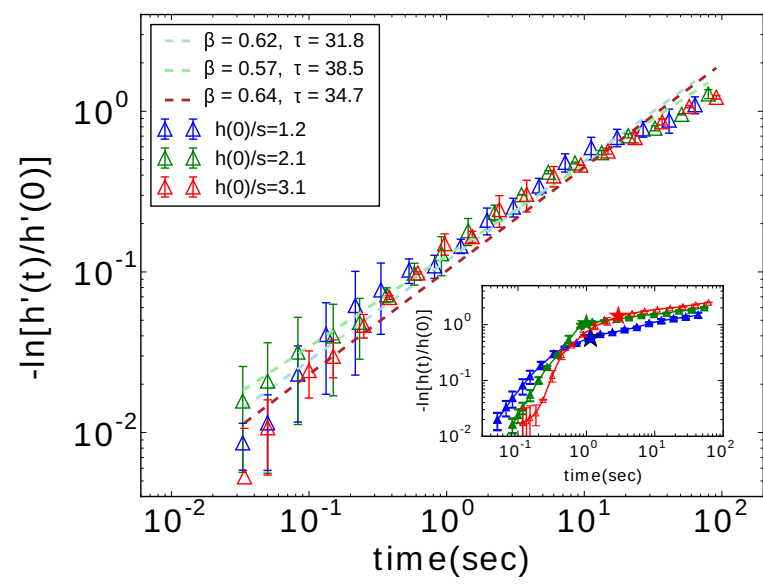

Figure 5. Main: Collapse dynamics after $T_{b}$ for HF hexapod on HF base. Each color of triangles represents one $h(0)$, as shown in the legend. Dashed lines indicate a stretched exponential relation. Insert: Collapse dynamics of these runs from the start of the sinusoidal vibration. The star markers indicate the choice of $T_{b}$, which is around time of the cone-shape piles form.

\section{Conclusions}

In this paper, we quantitatively investigated the collapse dynamics of columns of hexapods (star-shaped particles) under constant sinusoidal vibration. For short and flat columns made of high or low friction particles, the dynamics of the centroid heights of the columns can be well fitted to stretched exponential relation for different column diameters. We also find that the inter-particle friction increases the characteristic relaxation time. The collapse dynamics departs from a stretched exponential when the column is tall initially. A tall column undergoes a two-step process, where initially it collapses fast into a cone-shape pile, and afterwards its relaxation is described using a stretched exponential fit with similar parameters as the short and flat columns. We also investigate the effect of friction on the base that supports the column. High friction surfaces increase the characteristic relaxation time significantly.

\section{Acknowledgments}

We thank Dong Wang for useful discussions. Y.Z., and R.P.B. are funded by the U.S. National Science Foundation (grants NSF-DMR1206351 and DMS1248071),
NASA (grant NNX15AD38G) and the W. M. Keck Foundation. K.D. would like to thank the ITASCA Education Partnership Program (IEP).

\section{References}

[1] Liu, Andrea J., Nagel, Sidney R., Nature 396, 21 (1998)

[2] M. van Hecke, Journal of Physics: Condensed Matter 22, 033101 (2010)

[3] Bi, Dapeng, Jie, Zhang, Chakraborty, Bulbul, Behringer, Robert P., Nature 480, 355-358 (2011)

[4] C. Zhao, K. Tian, N. Xu, Phys. Rev. Lett. 106, 125503 (2011)

[5] A. G. Athanassiadis, M. Z. Miskin, P. Kaplan, N. Rodenberg, S. H. Lee, J. Merritt, E. Brown, J. Amend, H. Lipson, H. M. Jaeger, Soft Matter 10, 48 (2014)

[6] B. Saint-Cyr, J.-Y. Delenne, C. Voivret, F. Radjai, P. Sornay, Physical Review E 84, 041302 (2011)

[7] E. Brown, A. Nasto, A. G. Athanassiadis, H. M. Jaeger, Physical Review Letters 108, 108302 (2012)

[8] N. Gravish, S. V. Franklin, D. L. Hu, D. I. Goldman, Physical Review Letters 108, 208001 (2012)

[9] M. Trepanier, S. Franklin, Physical Review E 82, 011308 (2010)

[10] Y. Zhao, K. Liu, M. Zheng, J. Barés, K. Dierichs, A. Menges, R.P. Behringer, Granular Matter 18, 24 (2016)

[11] K.A. Murphy, N. Reiser, D. Choksy, C.E. Singer, H.M. Jaeger, Granular Matter 18, 26 (2016)

[12] Y. Jiao, F. H. Stillinger, S. Torquato, Physical Review E 79, 041309 (2009)

[13] J. de Graaf, R. van Roij, M. Dijkstra, Physical Review Letters 107, 155501 (2011)

[14] S. V. Franklin, Europhysics Letters 106, 58004 (2014)

[15] K. Desmond, S. Franklin, Physical Review E 73, 031306 (2006)

[16] S. Keller, H.M. Jaeger, Granular Matter 18, 29 (2016)

[17] K. Dierichs, A. Menges, Architectural Design 85, 86 (2015)

[18] M.Z. Miskin, H.M. Jaeger, Soft Matter 10, 3708 (2014)

[19] H.M. Jaeger, Soft Matter 11, 12 (2015)

[20] J.C. Phillips, Reports on Progress in Physics 59, 1133 (1996) 